\title{
Fuzzy logics with truth hedges revisited
}

\author{
Francesc Esteva, Lluís Godo, Carles Noguera \\ IIIA, Artificial Intelligence Research Institute \\ CSIC, Spanish National Research Council \\ Campus de la Univ. Autònoma de Barcelona s/n \\ 08193 Bellaterra, Catalonia, Spain \\ \{esteva,godo,cnoguera\}@iiia.csic.es
}

\begin{abstract}
In this paper we build upon previous works of Hájek and Vychodil on the axiomatization of truthstressing and depressing hedges as expansions of BL logic by new unary connectives. They show that their logics are chain-complete, but standard completeness is only proved for the expansions over Gödel logic. We propose weaker axiomatizations that have as main advantages the preservation of standard completeness properties of the original logic and the fact that any subdiagonal (resp. superdiagonal) non-decreasing function on $[0,1]$ preserving 0 and 1 is a sound interpretation of the truth stresser (resp. depresser) connectives.
\end{abstract}

Keywords: Truth hedges, Mathematical Fuzzy Logic, Standard completeness, T-norm based logics.

\section{Introduction}

In this paper we deal with interpretation of linguistic hedges in the t-norm based fuzzy logics. Typical examples of linguistic hedges in the sense of Zadeh [1] are "very true", "quite true", "more or less true", "slightly true", etc. They are represented in fuzzy logic systems in broad sense as functions from the set of truth values (typically the real unit interval) into itself, that modify the meaning of a proposition by applying over the membership function of the fuzzy set underlying the proposition.

In order to cope with these linguistic hedges in the setting of mathematical fuzzy logic, Hájek proposes in a series of papers $[2,3,4]$ to understand them as truth functions of new unary connectives called truth-stressing or truth depressing hedges, depending on whether they reinforce or weaken the meaning of the proposition they apply over. The intuitive interpretation of a truth-stressing (resp. depressing) hedge like very true (resp. slightly true) on a chain of truth-values is a subdiagonal (resp. superdiagonal) non-decreasing function preserving 0 and 1 . The class of such functions will be called hedge functions from now on.

This paper builds upon previous works, mainly those by Hájek [4] and Vychodil [5], on the axiomatization of truth-stressing (resp. depressing) hedges as expansions of BL logic (and of some of their prominent extensions, like Łukasiewicz or Gödel logics) by a new unary connective $v t$, for very true, and another one st, for slightly true, respectively. The logics they define are shown to be algebraizable and to enjoy completeness with respect to the classes of chains of their corresponding varieties, however not any BL-chain expanded with such functions (called hedges from now on) are models of them, or in other words, belong to the corresponding varieties. Moreover, the defined logics are not proved to enjoy in general standard completeness, except for the case of logics over Gödel logic. One of the main reasons for both problems is the presence in the axiomatizations of the well-known modal axiom $\mathrm{K}$ for the $v t$ connective,

$$
v t(\varphi \rightarrow \psi) \rightarrow(v t \varphi \rightarrow v t \psi),
$$

which puts quite a lot of constraints on the hedges to be models of these logics with no natural algebraic interpretation.

Particular classes of truth-stressers have been also addressed in the literature. For instance, the wellknown globalization operator $\Delta$ (introduced independently by Monteiro in the context of intuitionistic logic [6] and by Baaz in the context of GödelDummett logics [7]) is a limit case of a truth stresser since, over a chain, it sends 1 to 1 and all the other elements to 0 , and the intuitive interpretation would be definitely true.

In this paper we propose weaker axiomatizations for both the truth-stressing and depressing connectives not imposing any constraint on hedges other than the ones we have mentioned above, and for which we can prove standard completeness.

The paper is structured as follows. In the next section we provide the necessary logical and algebraic preliminaries that will be used in the rest of the paper. In Section 3 we propose a general axiomatization for truth stressers while Section 4 focuses on truth depressers. Related work is discussed in Section 5 and we conclude with some final remarks in Section 6.

\section{Preliminaries}

In this section we gather from [8,9] some necessary results we will use in sections 3 and 4 . 
Let $\mathrm{L}$ be a finitary logic in a language $\mathcal{L}$. We say that L is a Rasiowa-implicative logic (c.f. [10]) if there is a binary (either primitive or definable by a formula) connective $\rightarrow$ of its language such that:

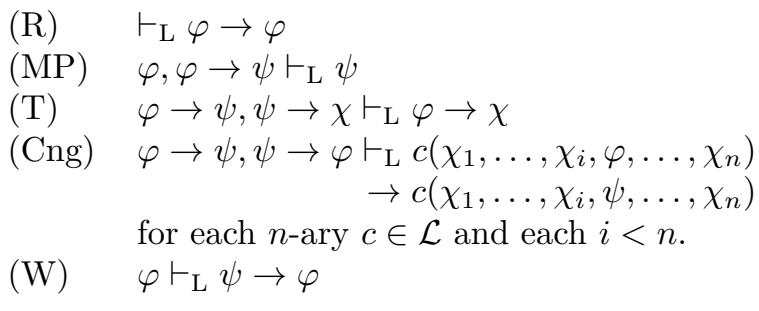

As proved in [8], every Rasiowa implicative logic is algebraizable in the sense of Blok and Pigozzi [11] and, if it is finitary, its equivalent algebraic semantics, the class of L-algebras, is a quasivariety; call it $\mathbb{L}$. Every L-algebra satisfies $x \rightarrow x=y \rightarrow y$ for any $x, y$, and hence the language can be expanded by a definable constant $\overline{1}=p \rightarrow p$. Then, the algebraizability gives the following strong completeness theorem:

For every set $\Gamma \cup\{\varphi\}$ of formulae, $\Gamma \vdash_{\mathrm{L}} \varphi$ iff for every $\boldsymbol{A} \in \mathbb{L}$ and every $\boldsymbol{A}$-evaluation e, $e(\varphi)=$ $\overline{1}^{\boldsymbol{A}}$, whenever $e[\Gamma] \subseteq\left\{\overline{1}^{\boldsymbol{A}}\right\}$.

Every L-algebra $\boldsymbol{A}$ is naturally endowed with a preorder relation by setting for every $a, b \in A: a \leq^{\boldsymbol{A}} b$ iff $a \rightarrow^{\boldsymbol{A}} b=\overline{1}^{\boldsymbol{A}}$. $\boldsymbol{A}$ is called an L-chain if $\leq^{\boldsymbol{A}}$ is a total $\operatorname{order}^{1}$. L is called a semilinear logic iff it is strongly complete with respect to the semantics given by L-chains.

Many systems informally referred to as fuzzy log$i c s$ in the literature are actually finitary Rasiowaimplicative semilinear logics. Well known examples are the three main fuzzy logics $G, \Pi$ and $€$ (see e.g. [12]), BL (the logic of all continuous tnorms, see $[12,13])$ and MTL (the logic of all leftcontinuous t-norms, see $[14,15])$. A big class of fuzzy logics which are finitary, Rasiowa-implicative and semilinear, and contain the mentioned prominent examples, are the so-called core fuzzy logics: the axiomatic expansions of MTL satisfying (Cng) for any possible new connective.

Finally, we need to recall a couple of definitions and results about disjunction connectives, for they provide a useful characterization of semilinearity.

A (primitive or definable) binary connective $\vee$ is called a disjunction in $\mathrm{L}$ whenever it satisfies:

$$
\begin{array}{ll}
(\mathrm{PD}) & \varphi \vdash_{\mathrm{L}} \varphi \vee \psi \quad \text { and } \quad \psi \vdash_{\mathrm{L}} \varphi \vee \psi \\
(\mathrm{PCP}) & \text { If } \Gamma, \varphi \vdash_{\mathrm{L}} \chi \text { and } \Gamma, \psi \vdash_{\mathrm{L}} \chi \text {, then } \\
& \Gamma, \varphi \vee \psi \vdash_{\mathrm{L}} \chi
\end{array}
$$

Given a disjunction $\vee$ and an a finitary inference rule $(R): \Gamma \vdash \varphi$ (axioms are taken as rules with $\Gamma=\emptyset)$, we define the $\vee$-form of $(R)$, denoted as $\left(R^{\vee}\right)$, as the rule $\Gamma \vee p \vdash \varphi \vee p$, where $p$ is an arbitrary propositional variable not appearing in $\Gamma \cup\{\varphi\}$.

\footnotetext{
${ }^{1}$ For the sake of a lighter notation, from now on we will drop the super-indexes in the algebra operations whenever no confusion is possible.
}

Proposition 1. [9] Let $\mathrm{L}_{1}$ be a logic with a disjunction $\vee$ and let $\mathrm{L}_{2}$ be an expansion of $\mathrm{L}_{1}$ by a set of finitary rules $\mathcal{C}$. Then, $\vee$ is a disjunction in $\mathrm{L}_{2}$ iff $\left(R^{\vee}\right)$ holds in $\mathrm{L}_{2}$ for each $(R) \in \mathcal{C}$. In particular, $\vee$ is a disjunction in any axiomatic expansion of $\mathrm{L}_{1}$.

Proposition 2. [9] Let L be a finitary Rasiowaimplicative logic with a binary connective $\vee$ satisfying (PD). Consider the following two properties:

$$
\begin{array}{ll}
\left(\mathrm{P}_{\vee}\right) & \vdash_{\mathrm{L}}(\varphi \rightarrow \psi) \vee(\psi \rightarrow \varphi) \\
\left(\mathrm{MP}_{\vee}\right) & \varphi \rightarrow \psi, \varphi \vee \psi \vdash_{\mathrm{L}} \psi \text { and } \\
& \varphi \rightarrow \psi, \psi \vee \varphi \vdash_{\mathrm{L}} \psi
\end{array}
$$

The following are equivalent:

(i) $\vee$ is a disjunction and satisfies $\left(\mathrm{P}_{\vee}\right)$,

(ii) $\mathrm{L}$ is semilinear and satisfies $\left(\mathrm{MP}_{\vee}\right)$.

As mentioned before, core fuzzy logics are semilinear, hence they are strongly complete with respect the class of their chains. However, this completeness may be sometimes refined to special subclasses of chains. We will use the following notions of completeness with respect to a given class of chains.

Definition $3(\mathbb{K C}, \mathrm{FS} \mathbb{K C}, \mathrm{S} \mathbb{K C})$. Let L be a core fuzzy logic and let $\mathbb{K}$ be a class of L-chains. We say that $\mathrm{L}$ has the (finitely) strong $\mathbb{K}$-completeness property, (F)SKC for short, when for every (finite) set of formulae $T$ and every formula $\varphi$ it holds that $T \vdash_{\mathrm{L}} \varphi$ iff $e(\varphi)=\overline{1}^{\boldsymbol{A}}$ for each $\boldsymbol{A}$-evaluation such that $e[T] \subseteq\left\{\overline{1}^{\boldsymbol{A}}\right\}$ for every L-algebra $\boldsymbol{A} \in \mathbb{K}$. We say that $\mathrm{L}$ has the $\mathbb{K}$-completeness property, $\mathbb{K C}$ for short, when the equivalence is true for $T=\emptyset$.

Of course, the $\mathrm{SKC}$ implies the FSKC, and the FSIKC implies the $\mathbb{K C}$. When $\mathbb{K}$ is the class of all chains whose support is the real unit interval $[0,1]$ we will denote it as $\mathcal{R}$, call its elements as real or standard chains, and we will speak about real or standard completeness properties.

\section{Truth stressers: a general axiomatization}

In order to solve the problems with the axiomatization of truth stressers and depressers proposed by Hájek and Vychodil mentioned in the Introduction section, in what follows we will make use of available results decribed in Section 2 to obtain a very simple and general axiomatizations with very intuitive properties and nice completeness results. To begin with let us consider the case of truth stressers.

Let $\mathrm{L}$ be a core fuzzy logic, and consider $\mathrm{L}_{s}$ the expansion of $\mathrm{L}$ with a new unary connective $s$ (for stresser) defined by the following additional axioms:

$$
\begin{aligned}
& \text { (VTL1) } s \varphi \rightarrow \varphi, \\
& \text { (VTL2) } s \overline{1},
\end{aligned}
$$

and the following additional inference rule:

$$
(\mathrm{MON}) \text { from }(\varphi \rightarrow \psi) \vee \chi \text { infer }(s \varphi \rightarrow s \psi) \vee \chi
$$


If we denote by $\vdash_{\mathrm{L}_{s}}$ the notion of deduction defined as usual from the above axioms and rules, one can easily show the following provabilities.

Lemma 4. In $\mathrm{L}_{s}$ the following deductions are valid:

(i) $\vdash_{\mathrm{L}_{s}} \neg s \overline{0}$

(ii) $\varphi \rightarrow \psi \vdash_{\mathrm{L}_{s}} s \varphi \rightarrow s \psi$

(iii) $\psi \vdash_{\mathrm{L}_{s}} s \psi$

(iv) $s \varphi, \varphi \rightarrow \psi \vdash_{\mathrm{L}_{s}} s \psi$

Proof. (i) It follows directly from (VTL1) taking $\varphi=\overline{0}$.

(ii) It follows directly from (MON) taking $\chi=\overline{0}$.

(iii) It follows directly from (ii) taking $\varphi=\overline{1}$ and using (VTL2).

(iv) Very easy using (ii) and modus ponens.

Notice that (iv) is a kind of stronger version of the modus ponens rule: if $\varphi$ implies $\psi$, and $\varphi$ is very true, then one can derive that $\psi$ is very true as well.

On the other hand, (ii) shows that (Cng) is satisfied for the new unary connective too. Therefore, the $\operatorname{logic} \mathrm{L}_{s}$ is Rasiowa-implicative and its equivalent algebraic semantics is the class of $\mathrm{L}_{s^{-}}$ algebras. An algebra $\mathcal{A}=\langle A, \wedge, \vee, \&, \rightarrow, s, \overline{0}, \overline{1}\rangle$ of type $\langle 2,2,2,2,1,0,0\rangle$ is an $\mathrm{L}_{s}$-algebra if it is an $\mathrm{L}$ algebra expanded by a unary operator $s: A \rightarrow A$ (truth-stressing hedge) that satisfies, for all $x, y, z \in$ A,

$$
\begin{aligned}
& \text { (1) } s(\overline{1})=\overline{1} \\
& \text { (2) } s(x) \leq x \\
& \text { (3) if }(x \rightarrow y) \vee z=\overline{1} \text { then } \\
& \quad(s(x) \rightarrow s(y)) \vee z=\overline{1} .
\end{aligned}
$$

It is clear that the class of $\mathrm{L}_{s}$-algebras forms a quasivariety, call it $\mathbb{L}_{s}$. Notice that if $\langle A, \wedge, \vee, \&, \rightarrow, \overline{0}, \overline{1}\rangle$ is a totally ordered L-algebra and $s: A \rightarrow A$ is any non-decreasing mapping such that $s(\overline{1})=\overline{1}$ and $s(a) \leq a$ for any $a \in A$, then the expanded structure $\langle A, \wedge, \vee, \&, \rightarrow, s, \overline{0}, \overline{1}\rangle$ is an $\mathrm{L}_{s}$-chain ${ }^{2}$. In other words, in $\mathrm{L}_{s}$-chains the quasiequation (3) turns out to be equivalently expressed by this simplified form: if $x \rightarrow y=\overline{1}$ then $s(x) \rightarrow s(y)=\overline{1}$, and this condition simply expresses that $s$ is non-decreasing.

Moreover, since the rule (MON) is closed under $\checkmark$-forms, by Proposition 1 we know that $\vee$ keeps being a disjunction in the expanded logic. On the other hand, since $\left(\mathrm{P}_{\vee}\right)$ was already valid in $\mathrm{L}$, by Proposition 2 we obtain that $\mathrm{L}_{s}$ is also semilinear and hence it is complete with respect to the semantics of all $\mathrm{L}_{s}$-chains.

Theorem 1. $\mathrm{L}_{s}$ is complete with respect to the class of all $\mathrm{L}_{s}$-chains.

Corollary 5. In $\mathrm{L}_{s}$ the following deductions are valid:

\footnotetext{
${ }^{2}$ Observe that these three simple conditions required for $s$ would be not enough to define an $\mathrm{L}_{s}$-chain in case $\mathrm{L}_{s}$ would have been defined with the additional axiom $\mathrm{K}$ as in [4].
}

(v) $\vdash_{\mathrm{L}_{s}} s(\varphi \vee \psi) \leftrightarrow s \varphi \vee s \psi$

(vi) $\vdash_{\mathrm{L}_{s}} s(\varphi \wedge \psi) \leftrightarrow s \varphi \wedge s \psi$

Proof. Both properties are easily checked on $\mathrm{L}_{s^{-}}$ chains.

One might wonder whether the corresponding equation for the monotonicity of $s$ (i.e. $s(x \wedge y)=$ $s(x) \wedge s(y)$ or, equivalently, $s(x \vee y)=s(x) \vee s(y))$ may substitute the quasiequation (3) in the definition of $\mathrm{L}_{s}$-algebras. In other words, does the quasivariety $\mathbb{L}_{s}$ coincide with the variety $\mathbb{V}$ of expansions of L-algebras satisfying the equations (1), (2) and mononicity of $s$ ? The answer is negative as shown by the following example.

Example 1. If $\mathrm{L}$ is not classical propositional logic, then there must be an L-chain $\boldsymbol{A}$ with at least three elements on its domain. Take any $a \in A \backslash\left\{\overline{0}^{\boldsymbol{A}}, \overline{1}^{\boldsymbol{A}}\right\}$ and consider the direct product algebra $\boldsymbol{A} \times \boldsymbol{A}$ and expand it with a unary operator $s$ by putting $s(a, b)=\langle a \wedge b, a \wedge b\rangle$. An easy computation shows that, although s satisfies (1), (2) and monotonicity, the rule $(M O N)$ is not sound. Indeed, we have $(\langle\overline{1}, \overline{1}\rangle \rightarrow\langle\overline{1}, a\rangle) \vee\langle a, \overline{1}\rangle=\langle\overline{1}, a\rangle \vee\langle a, \overline{1}\rangle=\langle\overline{1}, \overline{1}\rangle$, while $(s(\overline{1}, \overline{1}) \rightarrow s(\overline{1}, a)) \vee\langle a, \overline{1}\rangle=(\langle\overline{1}, \overline{1}\rangle \rightarrow\langle a, a\rangle) \vee$ $\langle a, \overline{1}\rangle=\langle a, a\rangle \vee\langle a, \overline{1}\rangle=\langle a, \overline{1}\rangle \neq\langle\overline{1}, \overline{1}\rangle$.

Thus, $\mathbb{V}$ and $\mathbb{L}_{s}$ coincide over chains but they are different. While $\mathrm{L}_{s}$ is semilinear due to the rule (MON), the logic associated to $\mathbb{V}$ is not. This also shows that in the presentation of $\mathrm{L}_{s}(\mathrm{MON})$ cannot be substituted by the simpler rule: from $\varphi \rightarrow \psi$ infer $s \varphi \rightarrow s \psi$ (which, as we have just seen, is sound in $\mathrm{L}_{s}$-chains but not for all $\mathrm{L}_{s}$-algebras).

Similarly, inspired by the well-known presentation of logics with $\Delta$, one might also ask whether (MON) could be substituted by the globalization rule for $s$ : from $\varphi$ infer $s \varphi$. The answer is again negative.

Example 2. Consider the finite MTL-chain $\boldsymbol{C}$ defined over the domain $C=\{0,1,2,3,4,5\}$ with the natural order and the following monoidal operation:

\begin{tabular}{c|cccccc}
$\&$ & 0 & 1 & 2 & 3 & 4 & 5 \\
\hline 0 & 0 & 0 & 0 & 0 & 0 & 0 \\
1 & 0 & 1 & 1 & 1 & 1 & 1 \\
2 & 0 & 1 & 1 & 1 & 2 & 2 \\
3 & 0 & 1 & 1 & 1 & 2 & 3 \\
4 & 0 & 1 & 2 & 2 & 4 & 4 \\
5 & 0 & 1 & 2 & 3 & 4 & 5
\end{tabular}

Take the MTL-filter $F=\{4,5\}$ and the following unary operation s:

\begin{tabular}{c|c}
$x$ & $s(x)$ \\
\hline 0 & 0 \\
1 & 1 \\
2 & 1 \\
3 & 3 \\
4 & 4 \\
5 & 5
\end{tabular}


It is clear that $s$ is subdiagonal, sends the top element to itself and it is non-decreasing. Moreover for every $x \in F, s(x) \in F$, i.e. it is sound w.r.t. the globalization rule for $s$. However, it is not sound w.r.t. the rule $(\mathrm{MON})$. Indeed, $3 \rightarrow 2=4 \in F$, while $s(3) \rightarrow s(2)=3 \rightarrow 1=3 \notin F$.

We consider now the issue of completeness of $\mathrm{L}_{s}$ with respect to the distinguished semantics of $\mathrm{L}_{s^{-}}$ chains. One can prove that if $\mathrm{L}$ has the finite strong standard completeness property $(\mathrm{FS} \mathcal{R} C)$, then $\mathrm{L}_{s}$ has it as well. As usual, this can be done by showing that the class $\mathrm{L}_{s}$-chains is partially embeddable into the subclass of standard $\mathrm{L}_{s}$-chains.

Theorem 2 (Finite strong standard completeness). If $\mathrm{L}$ is a finite strong standard complete core fuzzy logic, then the logic $\mathrm{L}_{s}$ is finite strong standard complete as well.

Proof. Assume that $\mathrm{L}$ has the FSRC. Take any $\mathrm{L}_{s}$-chain $\boldsymbol{A}=\langle A, \wedge, \vee, \&, \rightarrow, s, \overline{0}, \overline{1}\rangle$ and let $B$ be a finite partial subalgebra of $\boldsymbol{A}$. We have to show that there exists a standard $\mathrm{L}_{s}$-chain $\langle[0,1], \wedge, \vee, *, \Rightarrow$ $\left., s^{\prime}, 0,1\right\rangle$ and a mapping $f: B \rightarrow[0,1]$ preserving the existing operations. By assumption, the $s$-free reduct of $\boldsymbol{A}$ is partially embeddable into a standard L-chain $\langle[0,1], \wedge, \vee, *, \Rightarrow, 0,1\rangle$. Denote this embedding by $f$ and consider any non-decreasing and subdiagonal function $s^{\prime}:[0,1] \rightarrow[0,1]$ satisfying $s^{\prime}(f(x))=f(s(x))$ for every $x \in B$ such that $s(x) \in B$. There are obviously many such functions $s^{\prime}$ interpolating the set of points $P=$ $\{\langle f(x), f(s(x))\rangle \mid x, s(x) \in B\}$, for instance a linear interpolant. Another interpolant can be defined as follows: let $0=z_{1}<\ldots<z_{n}<1$ be the set of elements of $[0,1]$ such that $\left\langle z_{i}, \cdot\right\rangle \in P$ and define $s^{\prime}(1)=1$ and, for all $z \in[0,1)$,

$$
s^{\prime}(z)=f\left(s\left(x_{i}\right)\right), \text { if } z_{i} \leq z<z_{i+1}
$$

where $x_{i} \in B$ is such that $z_{i}=f\left(x_{i}\right)$. In any case $s^{\prime}$ makes $\left\langle[0,1], \wedge, \vee, *, \Rightarrow, s^{\prime}, 0,1\right\rangle$ an $\mathrm{L}_{s}$-chain and $f$ a partial embedding of $\mathrm{L}_{s}$-chains.

Actuallly, this theorem can be generalized to arbitrary classes of L-chains and their $s$-expansions, proved in a completely analogous way, and yielding a more general result.

Corollary 6. Let L be a core fuzzy logic, $\mathbb{K}$ a class of $\mathrm{L}$-chains, and $\mathbb{K}_{s}$ the class of the $\mathrm{L}_{s}$-chains whose $s$-free reducts are in $\mathbb{K}$. If $\mathrm{L}$ has the $\mathrm{FS} \mathbb{K C}$.

Theorem 3 (Strong standard completeness). If $\mathrm{L}$ is a strong standard complete core fuzzy logic, then the logic $\mathrm{L}_{s}$ is strong standard complete as well.

Proof. Assume that L has the SRC. We have to show that any countable $\mathrm{L}_{s}$-chain can be embedded into a standard $\mathrm{L}_{s}$-chain. Let $\boldsymbol{A}$ be a countable $\mathrm{L}_{s}$-chain. By the assumption, the $s$-free reduct of $\boldsymbol{A}$ is embeddable into a standard L-chain $\boldsymbol{B}=$ $\langle[0,1], \wedge, \vee, *, \Rightarrow, 0,1\rangle$. Denote this embedding by $f$ and define $s^{\prime}: B \rightarrow B$ in the following way: for each $z \in[0,1], s^{\prime}(z)=\sup \{f(s(x)) \mid x \in A, f(x) \leq z\}$. So defined, $s^{\prime}$ is a non-decreasing and subdiagonal function such that $s^{\prime}(f(x))=f(s(x))$ for any $x \in A$ and hence $\boldsymbol{B}$ expanded with $s^{\prime}$ is a standard $\mathrm{L}_{s^{-}}$ chain where $\boldsymbol{A}$ is embedded.

Observe that the proof of the previous theorem can be repeated whenever the linear order of the chains is complete. Therefore we obtain the following corollary.

Corollary 7. Let L be a core fuzzy logic, $\mathbb{K}$ a class of completely ordered L-chains, and $\mathbb{K}_{s}$ the class of the $\mathrm{L}_{s}$-chains whose s-reducts are in $\mathbb{K}$. If $\mathrm{L}$ has the $\mathrm{S} \mathbb{K}$, then $\mathrm{L}_{s}$ has the $\mathrm{SK}_{s} \mathrm{C}$.

\section{The case of truth depressers}

Very similarly to the case of truth stressers, we can proceed to define an axiomatization for the case of truth depressers just by replacing axioms (VTL1) and (VTL2) with dual versions (STL1) and (STL2) (for slightly true). Namely, given a core fuzzy logic $\mathrm{L}$, we define $\mathrm{L}_{d}$ as the expansion of $\mathrm{L}$ with a new unary connective $d$, the following additional axioms

$$
\text { (STL1) } \varphi \rightarrow d \varphi
$$$$
\text { (STL2) } \neg d \overline{0} \text {, }
$$

and the following additional inference rule

$$
\text { (MON) from }(\varphi \rightarrow \psi) \vee \chi \text { infer }(d \varphi \rightarrow d \psi) \vee \chi .
$$

Being a kind of dual version of $\mathrm{L}_{s}$, many properties are proved in a completely analogous way:

Lemma 8. In $\mathrm{L}_{d}$ the following deductions are valid:

(i) $\vdash_{\mathrm{L}_{d}} d \overline{1}$

(ii) $\varphi \rightarrow \psi \vdash_{\mathrm{L}_{d}} d \varphi \rightarrow d \psi$

(iii) $\neg \varphi \vdash_{\mathrm{L}_{d}} \neg d \varphi$

(iv) $\vdash_{\mathrm{L}_{d}} \neg d \varphi \rightarrow \neg \varphi$

(v) $d \varphi, \varphi \rightarrow \psi \vdash_{\mathrm{L}_{m}} d \psi$

Proof. (i) It follows directly from (STL1) taking $\varphi=\overline{0}$

(ii) It follows directly from (MON) taking $\chi=\overline{0}$.

(iii) It follows from (ii) for $\psi=\overline{0}$ and (STL2).

(iv) It follows directly from (STL1) using the fact that $(\varphi \rightarrow \psi) \rightarrow(\neg \psi \rightarrow \neg \varphi)$ is derivable in MTL.

(v) Very easy using (ii) and modus ponens.

It is interesting to remark that (v) provides a kind of weaker or modified version of modus ponens with the truth-depresser: if $\varphi$ implies $\psi$, and $\varphi$ is slightly true, then one can derive that $\psi$ is slightly true as well.

Again, (ii) shows that the (Cng) condition is satisfied for the new unary connective too. Therefore, the $\operatorname{logic} \mathrm{L}_{d}$ is Rasiowa-implicative and its 
equivalent algebraic semantics is the class of $\mathrm{L}_{d^{-}}$ algebras. An algebra $\mathcal{A}=\langle A, \wedge, \vee, \&, \rightarrow, s, \overline{0}, 1\rangle$ of type $\langle 2,2,2,2,1,0,0\rangle$ is an $\mathrm{L}_{d}$-algebra if it is an $\mathrm{L}$ algebra expanded by a unary operator $d: A \rightarrow$ $A$ (truth-depressing hedge) that satisfies, for all $x, y, z \in A$,

$$
\begin{aligned}
& \left(1^{\prime}\right) d(0)=0 \\
& \left(2^{\prime}\right) x \leq d(x), \\
& \left(3^{\prime}\right) \text { if } \quad(x \rightarrow y) \vee z=\overline{1} \text { then } \\
& \quad \quad(d(x) \rightarrow d(y)) \vee z=\overline{1} .
\end{aligned}
$$

Analogously to the case of truth-stressers, every Lchain $\boldsymbol{A}$ can be expanded to an $\mathrm{L}_{d}$-chain by adding an arbitrary non-decreasing mapping $d: A \rightarrow A$ such that $d(\overline{0})=\overline{0}$ and $x \leq d(x)$ for every $x \in A$.

Also, since the lattice disjunction keeps satisfying the $(\mathrm{PCP})$ in the expanded logic, $\mathrm{L}_{d}$ is semilinear and hence it is complete with respect to the semantics of all $\mathrm{L}_{d}$-chains. As a straightforward consequence, we have for $d$ an analogous result to Corolalry 5 for $s$

Lemma 9. In $\mathrm{L}_{d}$ the following deductions are valid:

$$
\text { (v) } \vdash_{\mathrm{L}_{d}} d(\varphi \vee \psi) \leftrightarrow d \varphi \vee d \psi
$$

(vi) $\vdash_{\mathrm{L}_{d}} d(\varphi \wedge \psi) \leftrightarrow d \varphi \wedge d \psi$

If we modify Example 1 by taking $s(a, b)=$ $\langle a \vee b, a \vee b\rangle$, it shows that in the context of truth depressers the rule (MON) cannot be substituted by simple monotonicity. Similarly, Example 2 can be modified by taking the function $\mathrm{d}$ :

\begin{tabular}{c|c}
$x$ & $d(x)$ \\
\hline 0 & 0 \\
1 & 1 \\
2 & 2 \\
3 & 4 \\
4 & 4 \\
5 & 5
\end{tabular}

and then it shows that in the presentation of $\mathrm{L}_{d}$ $(\mathrm{MON})$ cannot be substituted by the following rule: from $\neg \varphi$ infer $\neg d \varphi$. Indeed, $d$ is superdiagonal, sends the bottom element to itself and it is nondecreasing, it satisfies $\neg d(x) \in F$ whenever $\neg x \in F$; however, $3 \rightarrow 2=4 \in F$, while $d(3) \rightarrow d(2)=4 \rightarrow$ $2=3 \notin F$.

Finally, analogous proofs of those of Corollaries 6 and 7 allows us to obtain this theorem about the preservation of completeness properties.

Theorem 4 (Completeness properties). Let $\mathrm{L}$ be a core fuzzy logic, $\mathbb{K}$ a class of $\mathrm{L}$-chains and $\mathbb{K}_{d}$ the class of $\mathrm{L}_{d}$-chains whose d-free reducts are in $\mathbb{K}$. Then:

(i) If $\mathrm{L}$ has the $\mathrm{FSIKC}$, then $\mathrm{L}_{d}$ has the $\mathrm{FSK}_{d} \mathrm{C}$.

(ii) If $\mathrm{L}$ has the $\mathrm{S} \mathbb{K C}$ and all the chains in $\mathbb{K}$ are completely ordered, then $\mathrm{L}_{d}$ has the $\mathrm{SK}_{d} \mathrm{C}$.

\section{Related work}

As mentioned in the introduction, there are two main references when talking about the formalisation of truth-stressing hedges within the framework of mathematical fuzzy logic. The first one is Hájek's paper [4] where he axiomatizes over BL a logic for the hedge very true. The second one is the paper by Vychodil [5] where he extends Hájek's analysis to truth-depressing hedges. In the rest of this section we overview these and some related logics and compare them with our proposal.

\subsection{On Hájek's logic of "very true"}

Hájek defines the logic $\mathrm{BL}_{v t}$ as the expansion of $\mathrm{BL}$ with a new connective $v t$ and the following axioms

$$
\begin{aligned}
& (\mathrm{VE} 1) \text { vt } \varphi \rightarrow \varphi, \\
& (\mathrm{VE} 2) v t(\varphi \rightarrow \psi) \rightarrow(v t \varphi \rightarrow v t \psi), \\
& (\mathrm{VE} 3) v t(\varphi \vee \psi) \rightarrow(v t \varphi \vee v t \psi)
\end{aligned}
$$

and the following necessitation inference rule:

(NEC) necessitation for $v t$ : from $\varphi$ infer $v t \varphi$

If we define the corresponding logic $\mathrm{L}_{v t}$ with this kind of truth stresser over a core fuzzy logic L instead of BL (with the same axioms and rules), it turns out, by simple inspection, that $\mathrm{L}_{v t}$ can be obtained from the logic $\mathrm{L}_{s}$ by replacing the (MON) inference rule by the axiom (VE2) and the necessitation rule for $v t$ (NEC). In general, this makes $\mathrm{L}_{v t}$ a strictly stronger logic than $\mathrm{L}_{s}$. An exception is when $\mathrm{L}$ is Gödel logic, since in that case $\mathrm{G}_{v t}$ and $\mathrm{G}_{s}$ are equivalent. This is due to the fact that in $\mathrm{G}_{s}$ the axiom (VE2) is derivable.

Hájek proves completeness of $\mathrm{BL}_{v t}$ with respect to the class of linearly ordered $\mathrm{BL}_{v t}$-algebras, which extends to any axiomatic extension of $\mathrm{BL}$, but the issue of standard completeness is left as an open problem, except for the case of Gödel logic.

A relevant further study of logics with truth stressers can be found in the paper by Ciabattoni et al. [16], that makes significant contributions in various respects. They basically consider extensions of MTL as base logics to be expanded with a unary connective (called modality in [16]), they consider three possible additional axioms to be added to Hájek axiomatics, and they develop proof systems for the arising new logics and study their algebraic and completeness properties. Particularly relevant for our purposes, they consider for a given logic L that is an extension of MTL the following logics:

$$
\begin{aligned}
& \mathrm{L}-\mathrm{KT}^{r}=\mathrm{L}+(\mathrm{VE} 1)+(\mathrm{VE} 2)+(\mathrm{VE} 3)+(\mathrm{NEC}) \\
& \mathrm{L}-\mathrm{S} 4^{r}=\mathrm{L}-\mathrm{KT}^{r}+(\mathrm{VE} 4) v t \varphi \rightarrow v t(v t \varphi)
\end{aligned}
$$

Axiom (VE4), together with axiom (VE1) forces the truth-stressing hedges to be closed over their image, i.e. that $v t \varphi$ has to be equivalent to $v t(v t \varphi)$.

Notice that Hájek's $\mathrm{BL}_{v t}$ logic is nothing but the logic BL-KT ${ }^{r}$. Moreover, in their paper they prove 
standard completeness of the L-S4 ${ }^{r}$ logics for different choices for L: namely MTL, SMTL, $\mathrm{C}_{n} \mathrm{MTL}$, IMTL and $\mathrm{C}_{n}$ IMTL.

Other papers dealing with particular types of truth stressers are [2] where truth-stressers are closure operators while in $[3,17]$ where truth-stressers are further required to map any element to the greatest idempotent (w.r.t. to the monoidal operation) below it. Finally, observe that adding the axiom $v t \varphi \vee \neg v t \varphi$ to L-KT ${ }^{r}, v t$ turns to be equivalent to the well-know Baaz-Monteiro projection connective $\Delta$.

\subsection{Vychodil's logic of "slightly true"}

Vychodil first introduces in [5] a logic combining both a truth-streseer and a truth depresser. Indeed the logic $\mathrm{BL}_{v t, s t}$ is defined as an expansion of Hájek's $\mathrm{BL}_{v t}$ logic with a new unary connective "slightly true" denoted by st and with the following additional axioms

$$
\begin{aligned}
& \text { (ST1) } \varphi \rightarrow s t \varphi, \\
& \text { (ST2) st } \varphi \rightarrow \neg v t \neg \varphi, \\
& \text { (ST3) vt }(\varphi \rightarrow \psi) \rightarrow(\text { st } \varphi \rightarrow s t \psi)
\end{aligned}
$$

This logic is proved to be complete with respect to the class of all linearly-ordered $\mathrm{BL}_{v t, s t}$-algebras (defined in the obvious way). Note that axioms (ST1) and (ST2) put into relation both connectives $v t$ and st. Vychodil also proposes two slightly different axiomatizations (systems I and II) for the truth depressing hedge slightly true alone. They are defined again as expansions of BL with the unary connective st. Namely, system the (I) has the following set of additional axioms:

$$
\begin{aligned}
& (\mathrm{DH} 1) \varphi \rightarrow s t \varphi, \\
& (\mathrm{DH} 2) \neg \operatorname{st}(\overline{0}), \\
& (\mathrm{DH} 3) \operatorname{st}(\varphi \rightarrow \psi) \rightarrow(s t \varphi \rightarrow s t \psi)
\end{aligned}
$$

while the system (II) consists of the axioms (DH1), (DH2) and

$$
(\mathrm{DH} 4)(\varphi \rightarrow \psi) \rightarrow(s t \varphi \rightarrow s t \psi)
$$

Both systems also have the following rule of inference:

$$
\left(\mathrm{RN}_{s t}\right) \text { from } \neg \varphi \text { infer } \neg s t \varphi \text {. }
$$

Chain-completeness for both systems is proved, but again the issue of standard completeness is left open.

Notice that axioms (DH1) and (DH2) correspond exactly to (STL1) and (STL2) of our logic $\mathrm{L}_{d}$, and that the inference rule $\left(\mathrm{RN}_{s t}\right)$ is derivable from the rule (MON) using axiom (STL2). So, again, the main difference between Vychodil's logics and our proposal is the presence of the K-like axioms (DH3) and (DH4), which do not appear in the logics $\mathrm{L}_{d}$.

\section{Conclusions}

In this paper we have been concerned in providing new axiomatic systems $\mathrm{L}_{s}$ and $\mathrm{L}_{d}$ for the truth hedges very true and slightly true, by weakening the proposed systems by Hájek and Vychodil, and defined over any core fuzzy logic L. The main advantage of the proposed systems with respect to the previously ones is that we can show standard completeness, i.e. completeness with respect to the class of standard L-chains expanded by arbitrary (stressing and depressing) hedges. The price paid in this process is that the class of corresponding algebras cannot be shown in general to be a variety any longer, but only a quasi-variety. Actually it remains as an open problem to prove or disprove whether they form in fact a variety in the general case (for instance, for L being Gödel logic it is indeed a variety). An easy solution to get a variety would be to make use of the Baaz-Monteiro $\Delta$ operator. In case this operator is definable in $\mathrm{L}$, as it happens e.g. when $\mathrm{L}$ is the $n$-valued Łukasiewicz $\operatorname{logic} \mathrm{E}_{n}$ (where $\Delta \varphi$ is defined as $\varphi \& . \stackrel{n}{.} \& \varphi$ ) or when $\mathrm{L}$ is an extension of $\mathrm{SBL}_{\sim}$, the expansion of SBL with an involutive negation $\sim$ (where $\Delta \varphi$ is defined as $\neg \sim \varphi$ ), then the (MON) inference rule in $\mathrm{L}_{s}$ can equivalently be replaced by the axiom

$$
\left(\mathrm{MON}_{\Delta}\right) \Delta(\varphi \rightarrow \psi) \rightarrow(s \varphi \rightarrow s \psi)
$$

and analogously for the $d$ connective in $\mathrm{L}_{d}$. If $\Delta$ is not definable in $\mathrm{L}$, then we can expand the base $\operatorname{logic} \mathrm{L}$ with the operator $\Delta$ together with its usual set of axioms and necessitation rule (see e.g. [12]) and consider the logics $\mathrm{L}_{\Delta, s}$ and $\mathrm{L}_{\Delta, d}$ instead, where the $(\mathrm{MON})$ inference is again replaced by the above $\left(\mathrm{MON}_{\Delta}\right)$ axioms.

Finally, to study logics combining both kinds of hedges along the line proposed in this paper is also a matter of future research.

Acknowledgments The authors acknowledge partial support of the Spanish projects FFI2008-03126E/FILO, TIN2009-14704-C03-03 and TIN201020967-C04-01.

\section{References}

[1] Lotfi A. Zadeh. A fuzzy set theoretic interpretation of linguistic hedges. Journal of Cybernetics, 2(3):4-34, 1972.

[2] Petr Hájek and Dagmar Harmancová. A hedge for Gödel fuzzy logic. International Journal of Uncertainty, Fuzziness and Knowledge-Based Systems, 8(4):495-498, 2000.

[3] Petr Hájek. Some hedges for continuous t-norms logics. Neural Network World, 12(2):159-164, 2002.

[4] Petr Hájek. On very true. Fuzzy Sets and Systems, 124(3):329-333, 2001. 
[5] Vilém Vychodil. Truth-depressing hedges and BL-logic. Fuzzy Sets and Systems, 157(15):2074-2090, 2006.

[6] António A. Monteiro. Algebrés de Heyting symétriques. Portugaliae Mathematica, 39(14):1-239, 1980.

[7] Matthias Baaz. Infinite-valued Gödel logic with 0-1-projections and relativisations. In Petr Hájek, editor, Gödel'96: Logical Foundations of Mathematics, Computer Science, and Physics, volume 6 of Lecture Notes in Logic, pages 23-33. Springer-Verlag, Brno, 1996.

[8] Petr Cintula and Carles Noguera. Implicational (semilinear) logics I: Basic notions and hierarchy. Archive for Mathematical Logic, 49(4):417-446, 2010.

[9] Petr Cintula and Carles Noguera. A general framework for fuzzy logics. In Petr Cintula, Petr Hájek, and Carles Noguera, editors, Handbook of Mathematical Fuzzy Logic. College Publications, 201x. To appear.

[10] Helena Rasiowa. An Algebraic Approach to Non-Classical Logics. North-Holland, Amsterdam, 1974.

[11] Willem J. Blok and Don L. Pigozzi. Algebraizable Logics, volume 396 of Memoirs of the American Mathematical Society. American Mathematical Society, Providence, RI, 1989.

[12] Petr Hájek. Metamathematics of Fuzzy Logic, volume 4 of Trends in Logic. Kluwer, Dordrecht, 1998.

[13] Roberto Cignoli, Francesc Esteva, Lluís Godo, and Antoni Torrens. Basic fuzzy logic is the logic of continuous t-norms and their residua. Soft Computing, 4(2):106-112, 2000.

[14] Francesc Esteva and Lluís Godo. Monoidal t-norm based logic: Towards a logic for leftcontinuous t-norms. Fuzzy Sets and Systems, 124(3):271-288, 2001.

[15] Sándor Jenei and Franco Montagna. A proof of standard completeness for Esteva and Godo's logic MTL. Studia Logica, 70(2):183-192, 2002.

[16] Agata Ciabattoni, George Metcalfe, and Franco Montagna. Algebraic and prooftheoretic characterizations of truth stressers for MTL and its extensions. Fuzzy Sets and Systems, 161(3):369-389, 2010.

[17] Franco Montagna. Storage operators and multiplicative quantifiers in many-valued logics. Journal of Logic and Computation, 14(2):299322, 2004. 